\title{
Cambio de conducta activa de participantes del Programa Vida Sana
}

\author{
Active behaviour change of Vida Sana Program participants \\ * Rocío Bizama, ${ }^{* *}$ Misael Sandoval-Cárcamo, \& *** Marcelo Castillo-Retamal
}

Bizama, R., Sandoval-Cárcamo, M., \& Marcelo Castillo-Retamal, M. (2027). Cambio de conducta activa de participantes del Programa Vida Sana. Revista Ciencias de la Actividad Física UCM, 22(2), julio-diciembre, 1-12. http://doi.org/10.29035/rcaf.22.2.10

\section{RESUMEN}

El objetivo del presente estudio fue analizar la modificación de la conducta de los participantes del Programa Vida Sana, dirigido por el Ministerio de Salud de Chile (MINSAL) en relación a cuatro aspectos asociados al nivel de Actividad Física. Participaron 43 mujeres (entre 15 a 59 años) que ingresaron al programa que se realiza en un Centro de Salud Familiar (CESFAM) de la comuna de Chiguayante. Para el desarrollo del estudio se aplicó el Cuestionario Internacional del Actividad Física (IPAQ) en un pre y post test. El Grupo fue divido en cuatro subgrupos por rango etarios: Adolescentes 14 a 19 años, Jóvenes 20 a 29 años, Adulto-Joven 30 a 39 años y Adultos sobre 40 años. En base a los resultados obtenidos, estos arrojan un aumento significativo de los METS en los diferentes Dominios estudiados de cada categoría. Es decir, la conducta de los participantes, comparándolo con el pre y post test, manifestó un cambio conductual positivo en ellos al finalizar el programa.

Palabras clave: Actividad Física, Mujeres, Programa de Salud, Vida Sana, Cuestionario Internacional de Actividad Física.

\section{ABSTRACT}

The aim of this study is to analyze behavior modification of the Healthy Life Program participants, implemented by the Ministry of Health of Chile (MINSAL) related to four aspects associated with Physical Activity. Forty-three women participated (between 15 and 59 years old) who entered the program held in Family Health Center in Chiguayante County.

To develop the study, the International Physical Activity Questionnaire (IPAQ) was applied in a pre and post-test. The Group was divided into four subgroups by age range: Adolescents 14 to 19 years, Youth 20 to 29 years, Adult-Youth 30 to 39 years, and Adults over 40 years.

Based on the results obtained, these show a significant increase in METS in the different domains studied for each category. In other words, the participants' behavior, compared with the pre and post-test, showed a positive behavioral change.

Key words: Physical activity, Women, Health Program, Health life, International Physical Activity Questionnaire.

\footnotetext{
* Profesora de Educación Física/ Magíster en Actividad Física y Deporte. Cesfam La leonera/ Cesfam Pinares. Chiguayante, Chile. https://orcid.org/0000-0003-0950-2499 | rocbizama1985@gmail.com

** Profesor de Educación Física - Magíster en Actividad Física y Deporte. Colegio San Francisco de Talcahuano. Concepción, Chile. https://orcid.org/0000-0002-9611-7136 | misael.sandovalc@gmail.com

*** Doctor. Universidad Católica del Maule - Grupo de Estudio en Educación, Actividad Física y Salud, GEEAFyS. Talca, Chile. https://orcid.org/0000-0002-7482-1165 | mcastillo@ucm.cl
} 


\section{INTRODUCCIÓN}

El sedentarismo y la obesidad son padecimientos que afectan a gran parte de la población mundial, siendo tratados con diversas acciones donde se incluye la intervención con Actividad Física específica (Brito et al., 2019)

El abundante análisis epidemiológico que se lleva haciendo desde unas décadas atrás sobre la Actividad Física, ha alcanzado conclusiones muy uniformes acerca de los beneficios de su práctica regular, tanto físicos como psicológicos (Ramírez et al., 2018). De esta manera, la Actividad Física es un componente central de la prevención de enfermedades crónicas (Chapela \& Martinuzzi, 2018). A pesar de los beneficios demostrados, los indicadores de inactividad física y sus consecuencias siguen siendo alarmantes en las mujeres (González \& Rivas, 2018).

A lo anterior, se suman los cambios en los estereotipos de género en el consumo de tabaco y alcohol, su incorporación masiva al mercado laboral y el sedentarismo contribuyen a aumentar el riesgo de enfermedades crónicas y una disminución de su calidad de vida (Díaz et al., 2019). No obstante, la práctica de ejercicio incrementa la resistencia a la fatiga, reduce la ansiedad, la depresión, mejora la capacidad funcional, el sueño, ayuda a relajarse e incrementa las relaciones interpersonales (Barona et al., 2019).

Desde la Organización Mundial de la Salud (OMS) se ha establecido una serie de recomendaciones para todas las personas sobre Actividad Física, las que se especifican de acuerdo a la edad, el tipo de ejercicio y su duración e intensidad. En conjunto con otras instituciones a nivel internacional y nacional, recomiendan, para una buena salud, que las personas adultas realicen un mínimo de 30 minutos de Actividad Física moderada, al menos cinco días a la semana, y 60 minutos diarios de una intensidad moderada a intensa para niños y jóvenes (OMS, 2020). También se ha comprobado que la Actividad Física produce a nivel psicológico cambios positivos en la autopercepción, mejorando la autoconfianza y la percepción de la imagen corporal (Villalobos et al., 2019).

Hay programas que logran reducir la obesidad y los más eficaces son los que combinaron dieta y Ejercicio Físico, siendo más recomendables aquellos en los que se realizaba una combinación de entrenamiento aeróbico y de fuerza resistencia muscular (Fernández et al, 2019).

Los programas de actividad físico-recreativas asociados a tratamientos complementarios e integrales, permiten optimizar procesos de salud al integrar tratamientos pertenecientes a diversas ciencias tales como la psicología, la nutrición, la medicina general e integral, entre otras (Hernández et al., 2017).

Nuestro país no ha quedado ajeno a esta iniciativa de nivel mundial, por lo que la población tiene acceso a participar de un programa que cuenta con la orientación y apoyo profesional, el que tiene como principal objetivo generar un impacto en donde el individuo tenga el control de elegir actividades que sean beneficiosas para la salud personal y la de su familia o entorno, gestionado y administrado por el Servicio Nacional de Salud (MINSAL, 2015).

Durante los últimos años las políticas en salud pública han propuesto fomentar estilos de vida activos para la población (MINSAL, 2021). Esto, producto del aumento de enfermedades que surgen como consecuencia de factores de riesgo, como la mal nutrición por exceso y la inactividad física o sedentarismo, condición que se genera por una serie de factores ambientales, sociales y personales, asociados al exceso de uso de tecnología presente en la sociedad, jornadas laborales y académicas cada vez más extensas, elección de transportes de tipo pasivos, escaso tiempo destinado a la recreación, entre otros. Es por ello que desde el Ministerio de Salud (MINSAL) se crea un programa preventivo y con un enfoque interdisciplinario para reeducar y generar un impacto que ayude a disminuir las cifras 
relacionadas a enfermedades cardiovasculares y diabetes específicamente ya que estas enfermedades tienen la característica de ser prevenibles (MINSAL, 2008).

El presente estudio analizó el cambio de conducta, en relación a los cuatro dominios asociados al nivel de Actividad Física, post participación en el Programa Vida Sana, de un grupo de mujeres beneficiarias del sistema público de salud de la comuna de Chiguayante, Región del Biobío, Chile.

\section{METODOLOGÍA}

\section{Diseño}

El presente estudio considera un enfoque de investigación cuantitativo, con un diseño no experimental, de corte descriptivo trasversal, que evalúa en nivel de actividad física antes y después de la participación en el Programa Vida Sana.

\section{Participantes}

Usuarios del sistema público de salud, considerando una muestra final de 43 mujeres entre 15 y 59 años, participantes del Programa Vida Sana (PVS), de un Centro de Salud Familiar de la comuna de Chiguayante, Región del Biobío, Chile. Las actividades preponderantes de las participantes corresponden a estudios secundarios o superiores, labores de casa y prestación de servicios.

Los participantes, asistieron durante seis meses, con un total de 72 horas, en 6 meses, con frecuencia de 3 veces por semana).

Todos los sujetos participaron de forma voluntaria, consignado a partir de la firma del consentimiento informado por escrito, de acuerdo a las normativas éticas de la Declaración de Helsinki.

\section{Instrumentos}

Para la determinación del nivel de actividad física se utilizó el Cuestionario Internacional de Actividad Física (IPAQ) versión larga en español en modalidad entrevista, que identifica los puntos de corte de intensidad en METs/min/sem clasificándolos en baja (<600 METs/min/sem), moderada (600 - 1500METs/min/sem) y alta (>600 METs/min/sem) (Craig et al., 2003).

De acuerdo a las orientaciones de los mismos autores (Craig et al., 2003), se identifica el nivel de intensidad de acuerdo a la cantidad de METs involucrados en la actividad, considerando las actividades de moderada intensidad a aquellas que se encuentran entre 3 y 6 METs, así como las actividades de intensidad vigorosa a las que se encuentran por sobre los 6 METs

\section{Procedimientos}

Se realizó la medición del nivel de actividad física en la primera sesión de derivación (pre test), como parte del proceso de diagnóstico de los participantes del módulo de actividad física del PVS. La medición post se realizó un mes después de finalizada la participación en el ciclo formal del módulo de actividad física del PVS.

\section{Análisis estadístico}

Para el análisis de las características demográficas de la muestra se utilizó estadística descriptiva con medidas de tendencia central como la media y desviación estándar de las. Para la determinación del nivel de significancia, se utilizó t de Student, adoptándose diferencias significativas cuando el valor $\mathrm{p}<0,05$.

\section{RESULTADOS}

La muestra estuvo conformada por 43 participantes mujeres entre 15 y 59 años, la que se subdividió en cuatro categorías; adolescentes, jóvenes, adultojoven y adultos, las cuales se desempeñaban en actividades estudiantiles, domésticas o de servicios, con un nivel educacional (medio completo) residentes de la comuna de Chiguayante.

De acuerdo al nivel de AF Total se presentan los resultados obtenidos en porcentaje por los diferentes grupos, en el pre y pos test. Se observa una alta concentración de participantes en la 
categoría "Bajo" durante el pre test, quienes luego del pos test, pasan a distribuirse en los otros dos niveles "Medio" y "Alto". De los 43 sujetos, sólo uno quedó en nivel Bajo tras el post test. La concentración del nivel de AF en el pre test fue en las categorías baja y moderado, lo que se modifica en el post test hacia las categorías "moderadas" y "altas" (Tabla 2).

Tabla 1

Niveles de AF total en pre y pos test.

\begin{tabular}{lcccccccc}
\hline & \multicolumn{2}{c}{ BAJO } & \multicolumn{2}{c}{ MOD } & \multicolumn{2}{c}{ ALTO } & \multicolumn{2}{c}{ N por grupo } \\
& Pre & POS & Pre & POS & Pre & Pos & & \\
ADOL & 3 & 1 & 2 & 3 & 2 & 3 & 7 & $(16 \%)$ \\
\hline JUVEN & 1 & & 5 & 3 & 2 & 5 & 8 & $(19 \%)$ \\
ADUL-JOVEN & 2 & 5 & 1 & 3 & 9 & 10 & $(23 \%)$ \\
ADULTO & 3 & & 8 & 4 & 7 & 14 & 18 & $(42 \%)$ \\
N & 9 & 1 & 20 & 11 & 14 & 31 & 43 & \\
por Nivel & $(21 \%)$ & $(2 \%)$ & $(47 \%)$ & $(26 \%)$ & $(33 \%)$ & $(72 \%)$ & & \\
\hline
\end{tabular}

En cuanto a la prueba $p$, se evidencian diferencias significativas* en las Medias de ambas pruebas (Pre y Post Test), en las 43 personas evaluadas en el programa, tanto a nivel general como por dominio (Tabla 2).

Tabla 2

Media y Prueba p Pre y Post Test.

\begin{tabular}{lccc}
\hline \multicolumn{1}{c}{ DOMINIO } & $\begin{array}{c}\text { MEDIA PRE TEST } \\
\text { MET/sem (+DE) }\end{array}$ & $\begin{array}{c}\text { MEDIA POST TEST } \\
\text { MET/sem (+DE) }\end{array}$ & VALOR $p$ \\
\hline TRANSPORTE & $415,17(959,35)$ & $497,51(624,73)$ & 0,270 \\
\hline TRABAJO & $583,02(1486,14)$ & $490,17(969,97)$ & 0,320 \\
\hline DOMÉSTICO & $934,53(954,60)$ & $1216,93(1213,21)$ & $0,030^{*}$ \\
OCIO Y RECREACIÓN & $577,36(878,20)$ & $1557,89(1081,35)$ & $0,000^{*}$ \\
TIEMPO SENTADO & $1497,25(693,10)$ & $1100,58(677,65)$ & $0,000650^{*}$ \\
TOTAL & $2510,28(2732,56)$ & $3781,23(2388,61)$ & $0,002^{*}$ \\
\hline
\end{tabular}

\section{ANÁLISIS POR DOMINIO}

En el dominio de Transporte, todas las categorías aumentan luego de participar en el programa y lo arrojado en el post test, a excepción de la categoría adultos, en el que se evidencia una disminución en el post test para dicho dominio. 


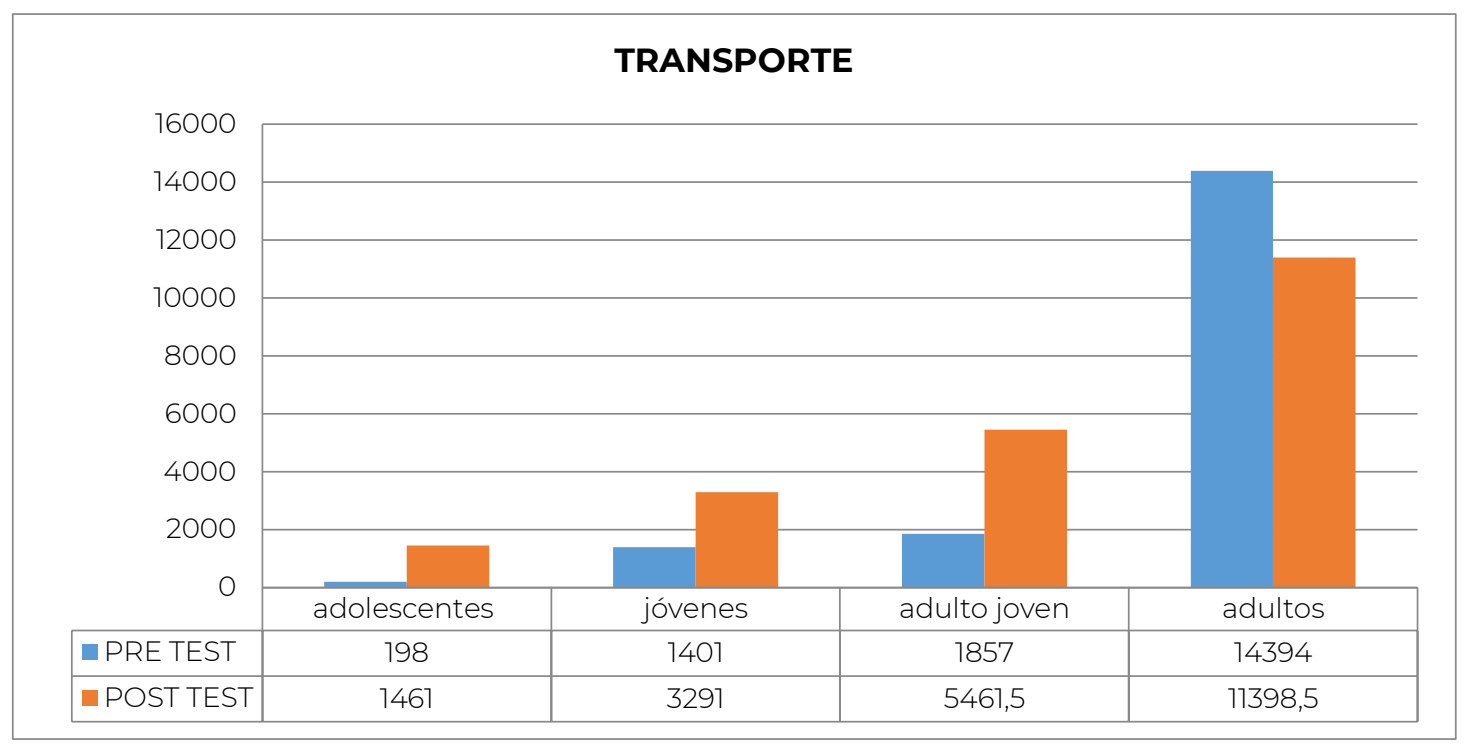

Figura 7. Transporte.

La figura evidencia un aumento del Dominio de Transporte en tres categorías, sobre el 50\%. Sólo en una categoría hay una disminución. Los cambios son más significativos en las tres categorías más jóvenes que en la categoría adultos.

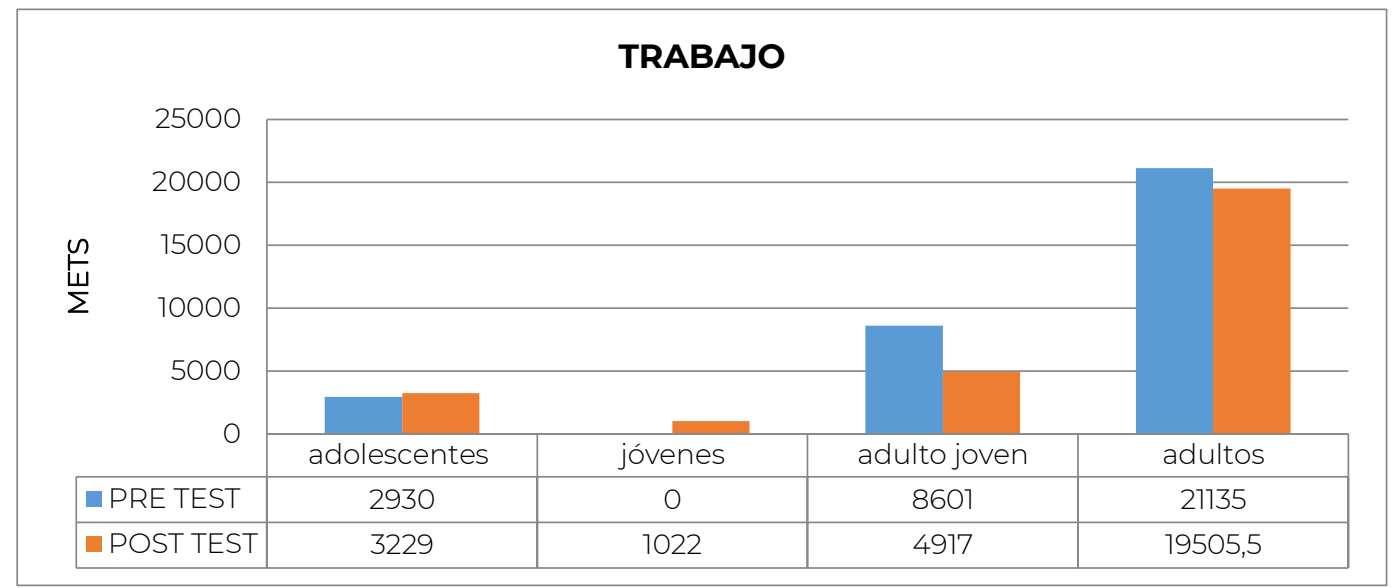

Figura 2. Trabajo.

De acuerdo a la Figura 2, dos categorías aumentan su nivel de AF en dicho Dominio y dos disminuyen levemente. Se evidencia un aumento de dicho Dominio en dos categorías por casi un 10\% y disminución en una. 


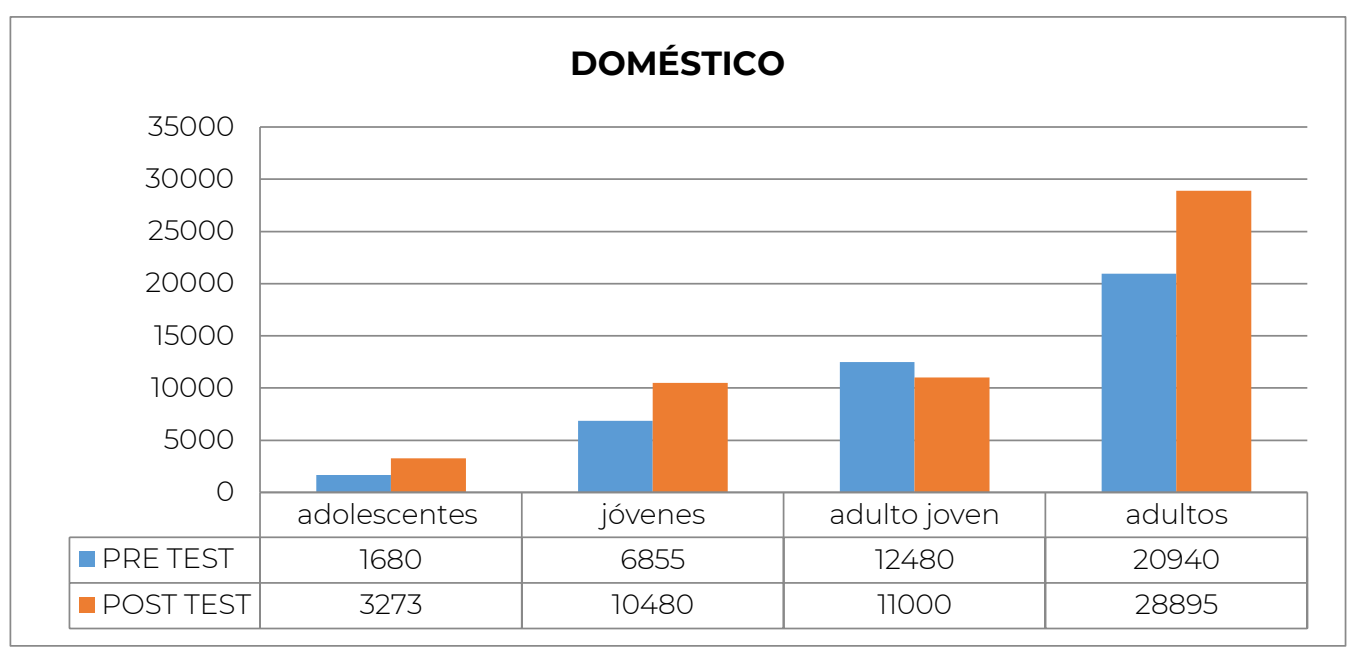

Figura 3. Doméstico.

La Figura 3, evidencia que casi todas las categorías aumentan sus MET después de participar en el programa. Sólo en la categoría Adulto joven, se evidencia una disminución en dicho dominio. Todas las categorías aumentan sobre un 20\%, a excepción de una (adulto-joven) que disminuyó sobre un 10\% (Tabla 6).

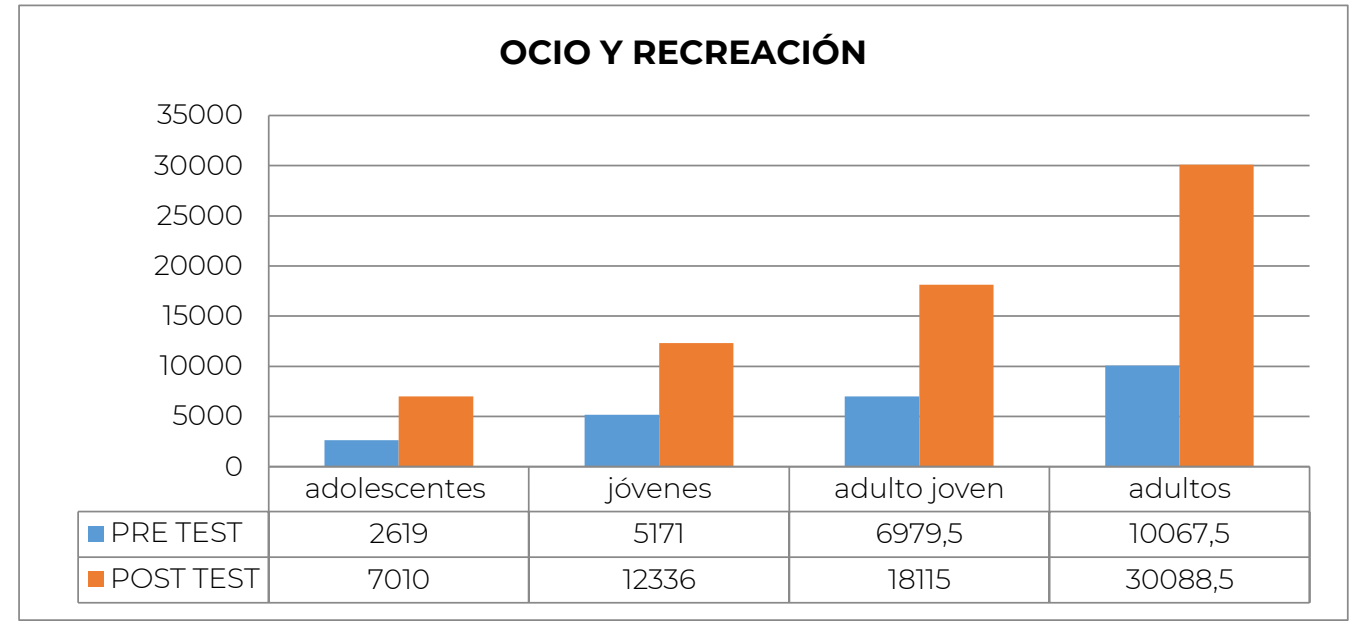

Figura 4. Ocio y Recreación.

En el Dominio Ocio y recreación, todas las categorías aumentan después de participar en el programa (Figura 4), en más de un 50\%. 


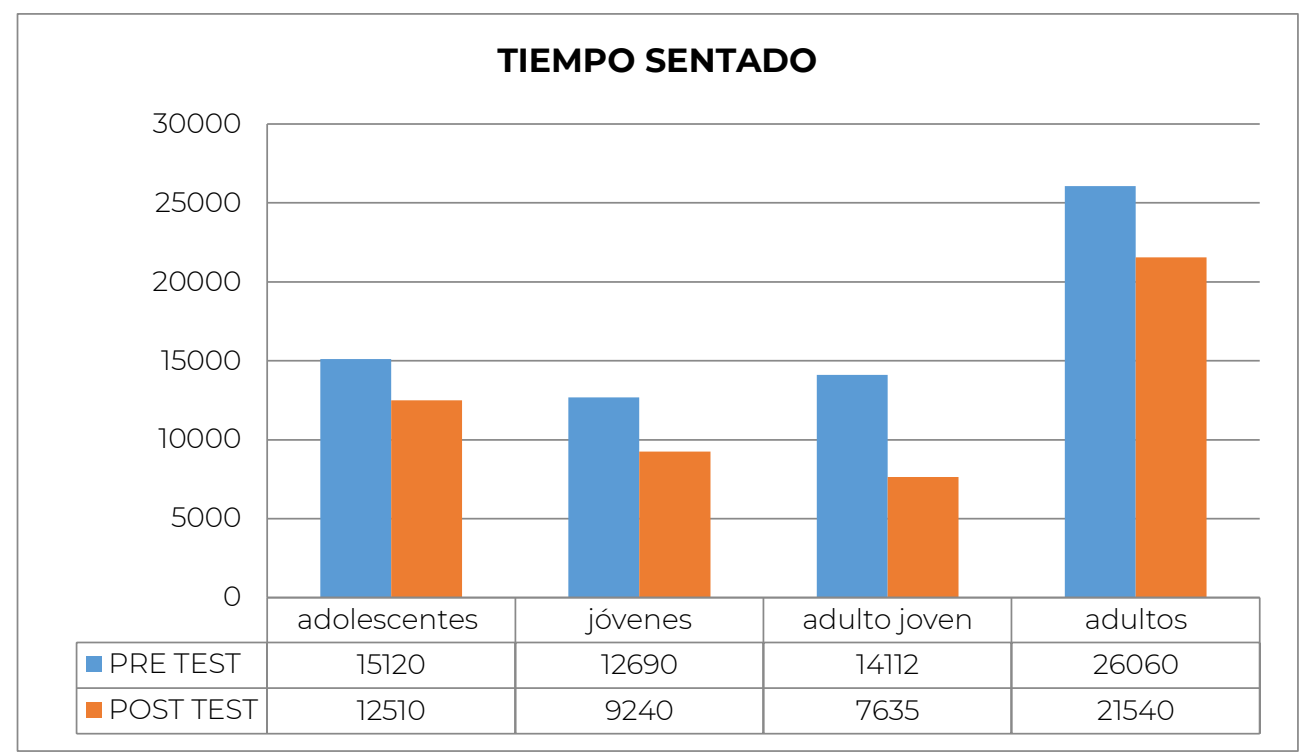

Figura 5. Tiempo sentado.

Conforme a lo expuesto en la Figura 5, se evidencia una disminución significativa del "tiempo sentado", luego de participar en el programa. En todas las categorías, se presenta una disminución sobre un $15 \%$ en el tiempo sentado.

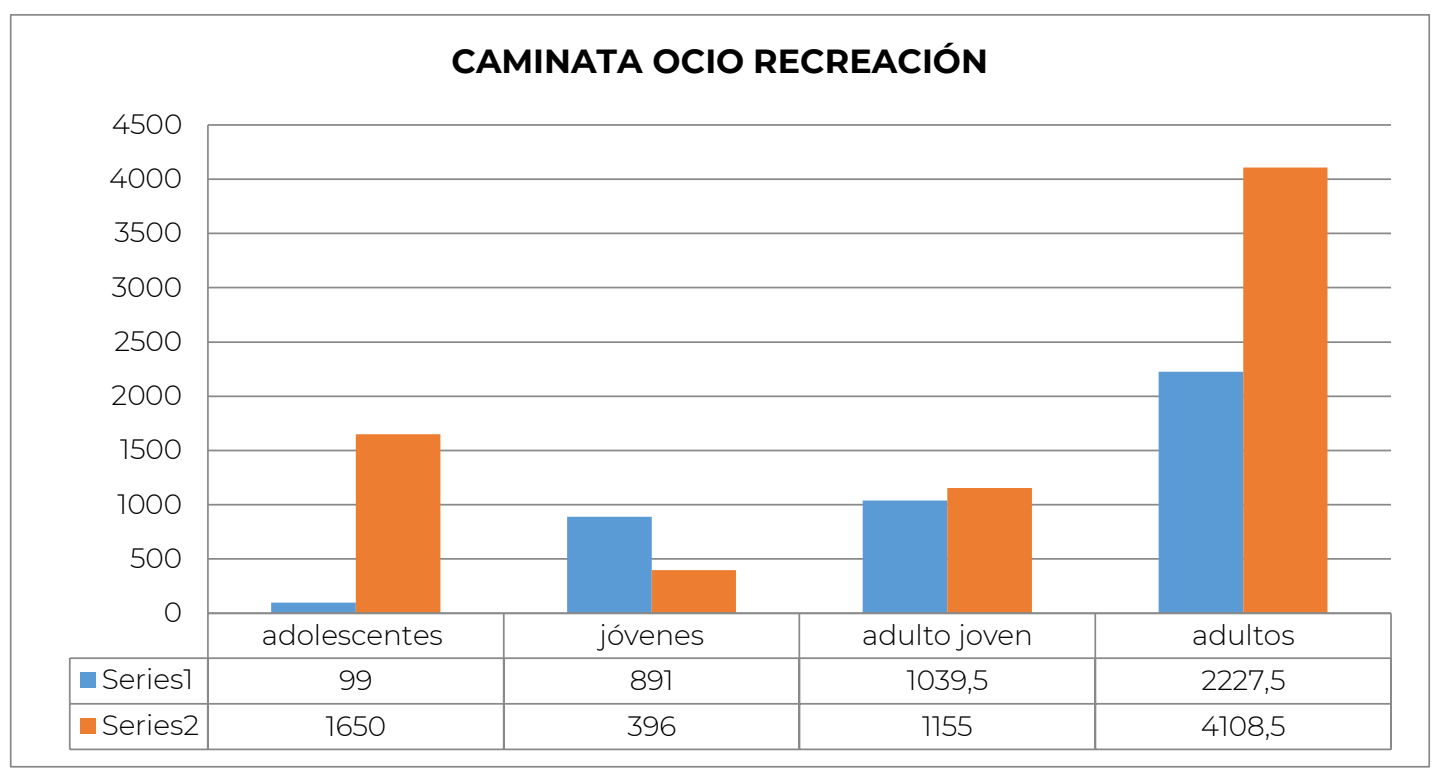

Figura 6. Caminata Ocio Recreación.

Casi todas las categorías aumentaron en el dominio Caminata Ocio Recreación, principalmente adolescentes y adultos. No obstante, la categoría de jóvenes, evidencia una disminución en más de la mitad entre el pre y pos test en dicho dominio. Si bien casi todas las categorías aumentan considerablemente, sólo la categoría "Jóvenes" presenta una disminución significativa comprándolo entre el pre y pos test en este Dominio (Figura 8). 


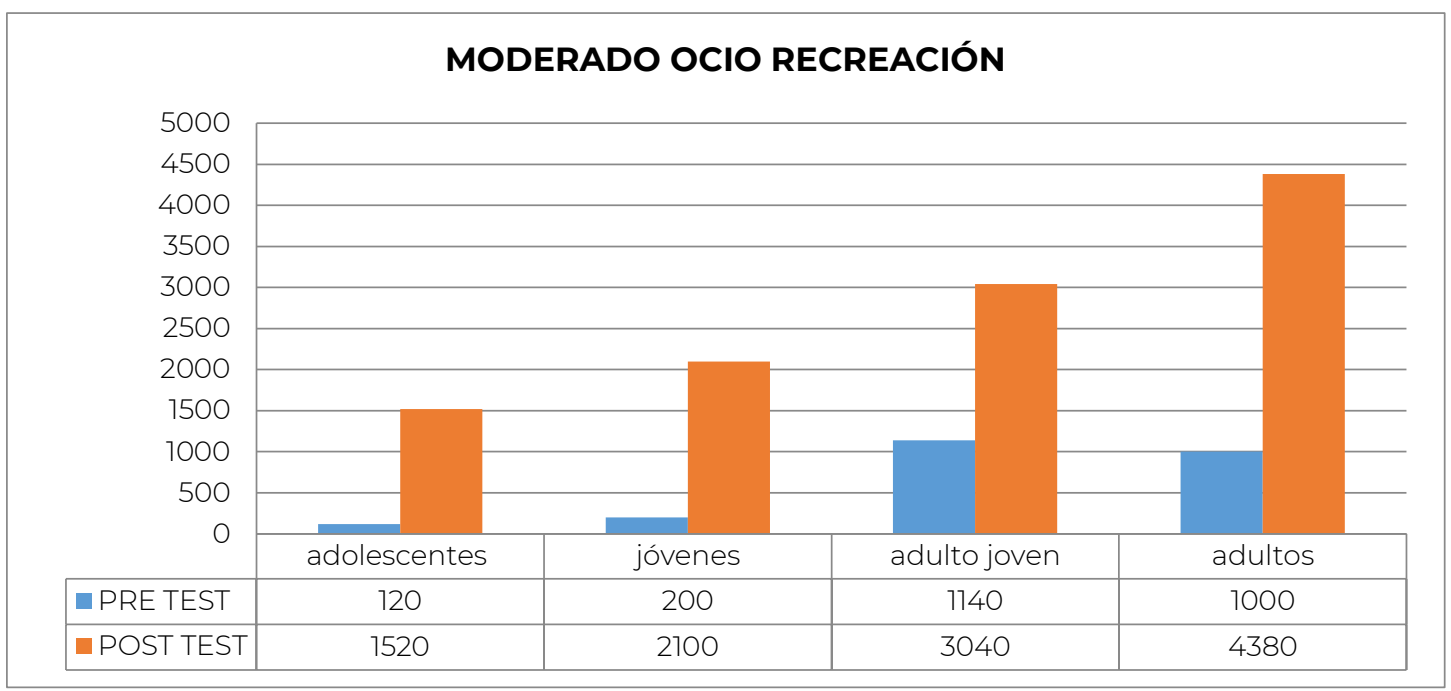

Figura 7. Moderado Ocio Recreación.

La Figura 7, demuestra un aumento muy significativo en dicho dominio, en todas las categorías evaluadas. Todas las categorías aumentan sobre un 60\% en el post test.

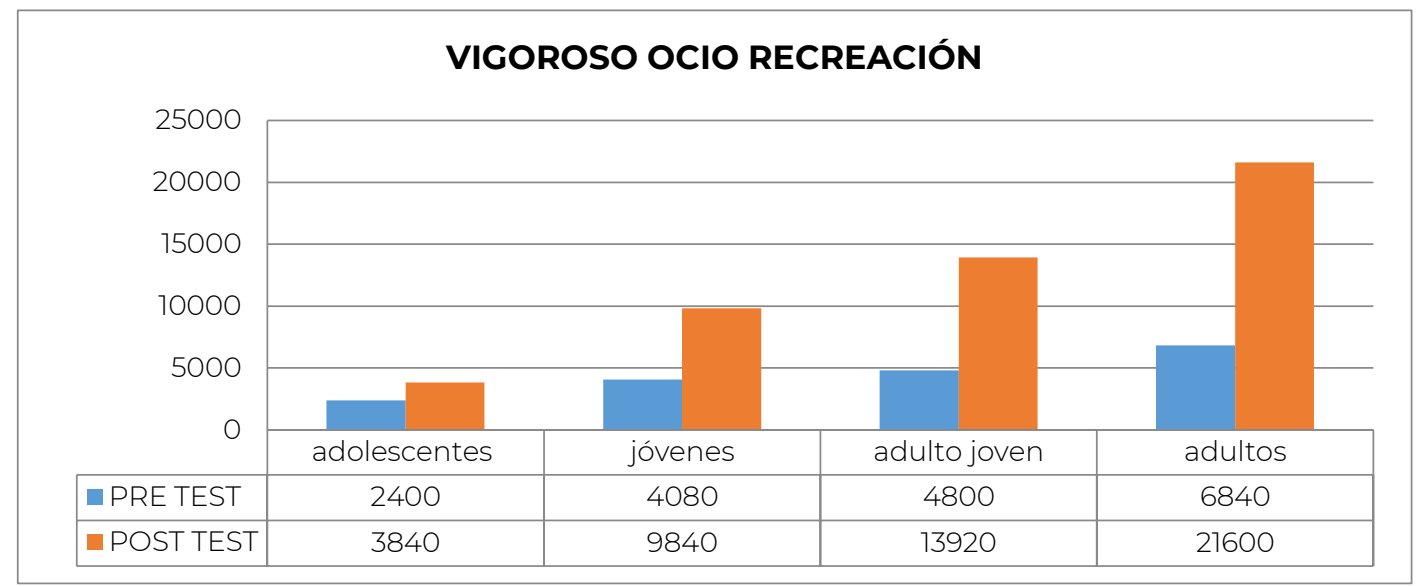

Figura 8. Vigoroso Ocio Recreación.

Todas las categorías aumentan sobre un 30\%, pero se evidencia un aumento significativo en las categorías Adulto Joven y Adultos. La categoría

\section{DISCUSIÓN}

Se observó una mejora en los cuatros dominios analizados en las participantes del programa de intervención, con una mejora considerable, en todas las edades. El dominio Transporte se mejora en un 50\% en relación a los datos del pre test. En el dominio Trabajo, la mejora es de un 10\%. En el
Adolescentes presenta un leve aumento en dicho Dominio.

dominio Doméstico, la variabilidad fue un alza entre el 10 y 20\%. Así como en el dominio Ocio y Recreación, el cambio fue positivo doblando el nivel de desempeño. 
De acuerdo a los resultados expuestos anteriormente, se observa una mejora en casi todos los dominios investigados. El dominio donde más se destaca este aumento, es en el de Ocio y Recreación, donde el resultado es similar al estudiado por Serón et al. (2010). Los autores mencionados, aplican el cuestionario a 1.535 sujetos, donde el 71,1\% son mujeres, con edades que oscilan entre los 35 y 70 años.

En cuanto al dominio de las Tareas del hogar, los investigadores antes mencionados (Serón et al., 2010), infieren que hay un bajo nivel de actividad, mientras que, en el presente estudio, los resultados se manifiestan a favor en los grupos adolescentes, jóvenes y adultos, en cambio, en el grupo adulto joven hay una leve disminución.

La investigación realizada por Chacón \& Contreras (2010), arroja que más de la mitad de las mujeres adultas, con edades de entre treinta y cinco a cincuenta y cuatro años, manifiestan un incremento de nivel de AF, en el Dominio de Transporte activo. Contrastando este último dato con la actual investigación, en ambos casos se aumenta en dicho Dominio. En las actividades Domésticas, en el primer estudio, todas aumentan su nivel de AF, mientras que, en el presente informe, los resultados arrojan datos similares en dicho Dominio, excepto en la categoría adulto joven que hay una leve disminución. En cuanto al tiempo libre, en el estudio de Chacón, más de la mitad incrementó su nivel de AF en lo que respecta al Dominio de tiempo libre, mientras que, en esta investigación, todas las categorías aumentan considerablemente.

El presente estudio, evidencia que el Dominio de "Transporte" es uno de los que menos desgaste energético produce. Pero el dominio de "Ocio" es el que presenta un mayor gasto energético en los participantes. En cuanto al dominio "Trabajo" coincide como otro dominio de bajo desgaste, pero el dominio "Doméstico" genera un alto desgaste. En un estudio realizado por Espínola \& Ramírez (2014), en donde se aplica el cuestionario a profesores y funcionarios universitarios, se evidenció que los dominios de Ocio y Transporte, eran los que generaban un menor desgaste energético en los participantes, en tanto, las más altas, en la presente investigación, son el dominio de "Trabajo" y "Doméstico".

En cuanto al género, se observa en este estudio que el $100 \%$ de los usuarios al programa son de sexo femenino, lo cual es concordante con la evaluación que se hace del mismo programa a través del Ministerio de Salud, manifestando como una debilidad que debe ser subsanada, la ausencia de un enfoque de género que incorpore a los hombres los cuales han sido casi inexistentes en la población beneficiaria adulta, en circunstancias que la población objetivo incluye a hombres y mujeres (en 2015 son $46 \%$ y $54 \%$ respectivamente). Otra debilidad que aparece en la evaluación del programa tiene relación con la deserción de beneficiarios que ocurre a nivel país y que fue en el año 2015 de un 86\%, lo que contrasta con este estudio ya que todos los participantes que ingresaron en el primer semestre terminaron un ciclo, incluso más de la mitad reingresó para un segundo periodo. No obstante, desde la evaluación que se hace es que se debe considerar que experiencias internacionales muestran que la deserción es un problema característico de los programas de este tipo (Daigre et al., 2016).

Como limitación del estudio, es que no se consideró un grupo aleatorio para el análisis, ni tampoco, variables indirectas que pudieran afectar el comportamiento de los participantes respecto de su nivel de práctica sistemática de actividad física, tales como: variables psicológicas, ambientales, nivel socioeconómico, nivel de escolaridad, por ejemplo.

\section{CONCLUSIÓN}

Los participantes evidencian un incremento importante de Actividad Física, luego de participar en el programa Vida Sana en un CESFAM de la comuna de Chiguayante. Es decir, al menos en ese recinto de salud, el programa es efectivo e incide positivamente en el cambio de conducta de los participantes, quienes reportaron cambios 
significativos en su nivel de AF, pasando de la categoría moderada a la categoría alta.

La concentración del nivel de AF en el pre test fue en las categorías baja y moderado, lo que se modifica en el post test hacia las categorías "moderadas" y "altas".

\section{REFERENCIAS BIBLIOGRÁFICAS}

Barona, P. A. C., López, V. C. L., Bustamente, A., Luna, E. C. W., \& Villani, G. P. V. (2019). Nivel de actividad física en mujeres con cáncer de mama no metastásico de dos instituciones de salud de alta complejidad. Revista Colombiana de Rehabilitación, 18(1), 42-52. https://doi.org/10.30788/RevColReh.v18.n1.2 019.298

Brito, L. P. Y., Hernández, P. C., Guarango, P. A. R., Reyes, Y. G., Tixi, M. E. G., \& Urquizo, Á. F. P. (2019). Efectos de un programa integral de actividad física para el personal docente y administrativo de salud. Revista Cubana de Medicina General Integral, 35(1). http://www.revmgi.sld.cu/index.php/mgi/ar ticle/view/994/242

Chacón Rivas, J. I., \& Contreras Hernández, I. A. (2010). Actividad física, consumo de oxígeno y características antropométricas en una población hipertensa femenina del Consultorio Barros LUCO. https://repositorio.uchile.cl/bitstream/hand le/2250/117460/Tesis\%20Javier\%20chac\%c3 $\%$ b3n\%20-

\%20Ignacio\%20Contreras.pdf?sequence=1 \&isAllowed =y

Chapela, S., \& Martinuzzi, A. (2018). Pérdida de masa muscular en el paciente críticamente enfermo: ¿Caquexia, sarcopenia y/o atrofia? Impacto en la respuesta terapéutica y la supervivencia. Revista Cubana de Alimentación y Nutrición, 28(2), 393-416. https://www.medigraphic.com/pdfs/revcub alnut/can-2018/can182k.pdf
Se sugiere, analizar otros centros donde se aplique el programa, lo cual permitirá una comparación de los resultados y diferenciarlos conforme al sector, población, tiempo de participación, rango etario, entre otros factores. El estudio puede ser ampliado a otras variables que intervienen en la conducta de los participantes y además ser extendidos a otros CESFAM.

Craig, C. L., Marshall, A. L., Sjöström, M., Bauman, A. E., Booth, M. L., Ainsworth, B. E., Pratt, M., Ekelund, U., Yngve, A., Sallis, J. F., \& Oja, P. (2003). International physical activity questionnaire: 12-country reliability and validity. Medicine \& science in sports \& exercise, 35(8), 1381-1395. https://doi.org/10.1249/01.mss.0000078924. 61453.fb

Daigre, M. L., Ferrer-Luies, M., \& Heynig Pepper, E. (2016). Resumen Ejecutivo: Evaluación Programas Gubernamentales (EPG) Programa Vida Sana Intervención en Factores de riesgo de enfermedades no transmisibles en niños, adolescentes, adultos y mujeres post parto. Ministerio de Salud Subsecretaría de Redes Asistenciales. https://www.dipres.gob.cl/597/articles149542_r_ejecutivo_institucional.pdf

Díaz, J. R., Fernández, C. A., Cabo, A. P., Pino, L. P., \& Gómez, C. A. (2019). Motivaciones y hábitos de actividad física en alumnos universitarios. Retos: nuevas tendencias en educación física, deporte y recreación, (36), 446-453. https://doi.org/10.47197/retos.v36i36.69906 
Espínola, Y. R., \& Ramírez Martínez, G. A. (2014). Aplicabilidad del cuestionario internacional de actividad física (IPAQ) en profesores y funcionarios de las facultades de la une de Minga Guazú. XXII Jornadas Jóvenes Investigadores, Valparaíso, Chile. http://ns2.une.edu.py:7004/repositorio/han dle/123456789/267

Fernández-Lázaro, D., Díaz, J., Caballero, A., \& Córdova, A. (2019). Entrenamiento de fuerza y resistencia en hipoxia: efecto en la hipertrofia muscular. Biomédica, 39(1), 212220.

https://doi.org/10.7705/biomedica.v39i1.408 4

González, N. F., \& Rivas, A. D. (2018). Actividad física y ejercicio en la mujer. Revista colombiana de cardiología, 25, 125-131. https://doi.org/10.1016/j.rccar.2017.12.008

Hernández Aguillar, B., Chávez Cevallos, E., de la Concepción Torres Marín, J., Torres Ramírez, A., \& Fleitas Díaz, I. M. (2017). Evaluación de un programa de actividad físico-recreativa para el bienestar físico-mental del adulto mayor. Revista Cubana de Investigaciones Biomédicas, 36(4),

1-16.

http://scielo.sld.cu/scielo.php?pid=S0864-

03002017000400007\&script=sci_arttext\&tl $n g=e n$

Ministerio de Salud (MINSAL). (2021). Orientaciones para la planificación y programación RED. Subsecretaría de Redes Asistenciales. https://www.minsal.cl/wp-

content/uploads/2020/09/20200908_ORIE NTACIONES-PARA-LA-PLANIFICACIONEN-RED-2027.pdf

Ministerio de Salud (MINSAL). (2015). Orientacionesy lineamientos Programa Vida Sana. Intervención en Factores de Riesgo de
Enfermedades No Transmisibles.

http://www.bibliotecaminsal.cl/wp/wp-

content/uploads/2018/01/015.OT-Vida-

Sana.pdf

Ministerio de Salud (MINSAL). (2008). Programa de Alimentación Saludable y Actividad Física para la Prevención de Enfermedades Crónicas en Niños, Niñas, Adolescentes y Adultos 2008-2009. https://www.minsal.cl/portal/url/item/69e15 fOc31354025e04001017f0133d1.pdf

Organización Mundial de la Salud (2020). Directrices de la OMS sobre actividad física y hábitos sedentarios: de un vistazo [WHO guidelines on physical activity and sedentary behaviour: at a glance]. https://apps.who.int/iris/bitstream/handle/1 0665/337004/9789240014817-spa.pdf

Ramírez Prieto, M. B., Raya Franco, M., \& Ruiz del Río, M. (2018). Sedentarismo y salud: efectos beneficiosos de la actividad física en estudiantes universitarios. ReiDoCrea, 7, 7984. https://doi.org/10.30827/Digibug.49826

Serón, P., Muñoz, S., \& Lanas, F. (2010). Nivel de actividad física medida a través del cuestionario internacional de actividad física en población chilena. Revista médica de Chile, 138(10), 1232-1239. http://dx.doi.org/10.4067/s003498872010001100004

Villalobos, F., Vinuesa, A., Pedret, R., Reche, A., Domínguez, E., \& Arija, V. (2019). Efecto de un Programa de actividad física sobre la autoestima en sujetos con enfermedades crónicas. Ensayo de intervención comunitaria «Pas a Pas». Atención Primaria, 57(4), 236-244. https://doi.org/10.1016/j.aprim.2017.11.011 


\section{Dirección para correspondencia}

Rocío Bizama Peña

Profesora de Educación Física/ Magíster en Actividad Física y Deporte.

Cesfam La leonera/ Cesfam Pinares.

Chiguayante, Chile.

Dirección Postal: Avda. 8 Oriente Edificio Sierra, dpto. 503

Condominio Alto Chiguayante,

Chiguayante, Biobío, Chile.

ORCID: https://orcid.org/0000-0003-0950-2499

Contacto:

rocbizama1985@gmail.com

Recibido: 18-02-2021

Aceptado: 01-03-2021 